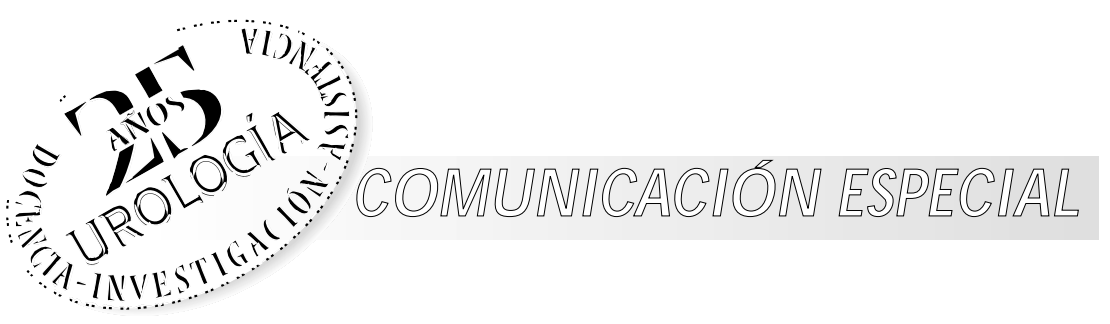

\title{
INTERNET EN MEDICINA ¿UNA AYUDA, UN PROBLEMA, UNA CAUSA DE ERRORES...?
}

\author{
F.J. SOLÉ BALCELLS \\ Servicio de Urología. Fundación Puigvert I.U.N.A. Barcelona. \\ Actas Urol Esp. 27 (3): 180-184, 2003
}

\section{RESUMEN}

“INTERNET EN MEDICINA ¿UNA AYUDA, UN PROBLEMA, UNA CAUSA DE ERRORES...?”

Internet ha significado para la Formación Médica Profesional un gran avance. Cada vez son más utilizadas las bases de datos de Medline en la Comunidad Científica. Los pacientes han empezado a usar Internet como fuente de información sobre sus dolencias, buscando a su través una segunda opinión. El artículo pone de manifiesto las ventajas de su uso para los propios enfermos, como asimismo el peligro que representa una información no bien comprendida o en ocasiones no válida científicamente. Las propias Asociaciones Urológicas deben responsabilizarse de las páginas web en forma clara, con datos de medicina basada en evidencia, informando a los pacientes que deseen confrontar la opinión de su especialista con la que se puede obtener por la red, siendo el propio médico, ante las personas que demuestren interés en ello, quien debe proporcionarles las direcciones web más idóneas.

PALABRAS CLAVE: Internet. Formación continuada. Peligrosidad de la información. Páginas web recomendables.

\section{ABSTRACT}

"CYBERMEDICINE: A HELP? A PROBLEM? AN ORIGIN OF ERRORS...?"

The Internet has been a breakthrough for Professional Medical Training. Medline databases are increasingly being used by the scientific community at large. Patients have also started to use the Internet as a source of information on their illnesses, and look in the web for a second opinion. This paper shows the advantages derived for the patients from using the Internet as well as the risks involved from a poorly understood, even not scientifically validated information. The Urology Associations themselves should take responsibility of the web sites in a unambiguous way, by including evidence-based medicine, offering assistance to those patients who want to compare their specialist's opinion with that obtainable in the Internet, and with the doctors themselved directing those who show a real interest to the most suitable web sites.

KEY WORDS: Continous training. Dangers from information. Recommended wed sites.

$\mathrm{E}^{\prime}$ lordenador fue utilizado por los médicos, en un principio, y en forma tímida, como procesador de textos, sustituyendo a la máquina de escribir. Fue después que los programas informáticos fueran utilizados para cálculos estadísticos, manejando las bases de datos de Internet a través de Medline. Es indiscutible que el orde- nador es básico para la actualización científica. Pero quizás lo que más ha motivado la utilización de Internet ha sido la posibilidad de obtener, tanto por profesionales médicos como por el público general, información, no siempre totalmente cierta, sobre enfermedades y su tratamiento ${ }^{1}$. 
Es imposible calcular en este momento cuantos millones de personas están "ligadas" a Internet. Sólo en Estados Unidos se consideraba hace un par de años que el número de "net-adictos" era superior a los 80 millones realizando un tercio de los americanos sus compras a través de la red $^{2}$. Es asimismo cada vez mayor el número de personas que leen las noticias del día en los periódicos virtuales, escuchan la radio o ven la televisión a través de ordenador, bajan música a través de Internet e incluso crean sus propios CD-musicales, estando en constante aumento el número de pacientes que consultan al médico un problema particular por este medio ${ }^{3}$ o bien leen las páginas dedicadas a la salud y a enfermedades específicas.

El paciente puede consultar de una forma totalmente gratuita la información sobre salud que existe en la red, muchas veces proporcionada por centros hospitalarios de prestigio ${ }^{4}$. El 90\% de los médicos de Canadá según informa el Dr. Jadad de la Universidad MacMaster de dicho país, han recibido alguna vez un paciente que les ha llevado información extraída de la red ${ }^{5}$.

Las tres cuartas partes de los canadienses que emplean Internet usan la web como fuente de información ante problemas de salud y dos de cada diez consultan las páginas sobre medicaciones en relación a su enfermedad. Brown señala que cerca de la mitad de los adultos que usan Internet han accedido en alguna ocasión a webs de consejo médico, y que ello representa en Estados Unidos, aproximadamente a más de 15 millones de personas ${ }^{6}$.

El problema de la utilización de Internet por los pacientes es la seguridad de que la información que reciben sea de suficiente calidad para seguirla como consejo. En un estudio realizado en una clínica ortopédica de Londres, en 363 pacientes que contestaron a un cuestionario sobre su proceso, el 52 por 100 informaron que habían buscado dicha información en la red, con mayor porcentaje a mayor edad y más alto nivel social. Un $25 \%$ contestaron que la información recibida les habían creado más confusiones que las que tenían previamente y un $20 \%$ dijeron que la información de Internet era totalmente distinta a la que les había proporcionado su especialista ${ }^{7}$.
Fue en 1997 cuando un artículo de Impicciatore $^{8}$, aparecido en el $\mathrm{BMJ}$, abrió la caja de Pandora al señalar a Internet como una fuente de información no totalmente fiable para los pacientes. Desde entonces han aparecido muchas revisiones y artículos que han mostrado su reserva sobre la calidad de la información médica que proporciona Internet y/o la televisión ${ }^{9,10}$. Su artículo comparaba las recomendaciones "on line" con las de los libros de texto, mostrando que raramente eran coincidentes.

El gran peligro de la información médica a través de Internet es la falta de control sobre la veracidad científica. En efecto, así como las revistas médicas poseen un comité editorial, con diferentes especialistas que analizan los artículos remitidos a la de revista, controlando la calidad de los ensayos randomizados, no existe ningún control en la red por lo que la mejor solución es aceptar solamente aquella información que provenga de "fuentes fiables"11.

Aunque es admisible, en una época en que el motor o energía de nuestro sistema es el dinero, que la única forma de mantener libre, es decir sin pago, la información en Internet es a través de anuncios de productos sanitarios farmacéuticos, la ética obligaría a que existiera una total separación entre el contenido científico y la información comercial, de forma y manera que los anunciantes no pudieran influir en la información de la página científica, e incluso sería conveniente que nunca el anuncio sobre un producto coincidiera en el tiempo y ubicación con una información científica sobre esta medicación. En una palabra, la ética de la información sanitaria debe respetarse en la red, distinguiéndose con claridad lo que es información científica y lo que es publicidad ${ }^{12}$.

Otra utilización de Internet por los pacientes o grupos interesados en los temas de salud es la práctica de conversaciones "on line", donde el sujeto expone sus experiencias, a veces en beneficio de otros pacientes, pero en ocasiones con argumentos contra la actuación médica que se le ha aplicado. Existen grupos que establecen una relación personal sobre temas específicos, como por ejemplo Asociaciones sobre la cistitis intersticial, el sida, etc. ${ }^{13}$. Nadie puede controlar la veracidad de estas revelaciones y lógicamente pueden dar lugar a grandes confusiones y rechazos fren- 
te a terapéuticas eficaces. El uso de la video-conferencia a nivel particular aún refuerza el efecto positivo o negativo de estos chats ${ }^{14}$. Aún más, el parecer del paciente, muchas veces erróneo frente a la actuación profesional del especialista, puede llevar a acusarle de mala práctica, siempre con el velo de la incógnita del acusador o informante.

El secreto profesional tiene que ver con la información que el médico da sobre pacientes a terceras personas pero no existe el punto contrario, el de la valoración del médico por el enfermo, ni existe regla ética que impida que el paciente explique o comente su versión de los hechos. Internet ofrece acceso anónimo a una gran cantidad de información sobre enfermedades y asimismo da soporte a grupos de las más raras enfermedades y estos pacientes, algunos -sólo supuestamente enfermos-, en vez de ir a un hospital, pueden consultar una tras otra infinidad de páginas web ${ }^{15}$.

Ante la peligrosidad del uso indiscriminado de Internet por los pacientes el médico debe, -si considera que el paciente es un ciberadicto-, proporcionarle las direcciones de páginas médicas fiables sobre su afección, para que les consulte, y gracias a ello obtenga un beneficio terapéutico.

$\mathrm{El}$ médico ignora en muchas ocasiones el sentir del paciente frente a su enfermedad, sin tener en cuenta sus comentarios o explicaciones. La lectura de las páginas web que los pacientes publican debe hacernos meditar sobre la relación médico-paciente. Un artículo del $\mathrm{BMJ}^{16}$ remarca la opinión de un paciente con esta sentida frase: "siempre hay una persona alrededor de un tumor".

Algunas publicaciones, -la revista JAMA ha sido la primera-, incluyen la llamada "página del paciente" para permitir saber y conocer las opiniones de los enfermos y gracias a ello mejorar la relación médico-paciente ${ }^{17}$.

Se están prodigando Guías de interés médico para pacientes para poder permitirles "navegar" a través de más de 7 millones de páginas con información sobre salud, señalando dichas Guías el indice de fiabilidad y seguridad de dichas publicaciones. Estas guías deben evitar crear "cibercondríacos", y que el paciente sustituya al consejo de su equipo médico por la información de la red. Lo que se pretende es tener al público más informado y que los médicos comprendan que el paciente precisa de razonamiento, consejos, información y atención humanizada. Es en este último punto que nunca Internet podrá sustituir la mirada, el calor humano y el razonamiento meditado por un buen profesional.

La American Medical Association ha editado Guías para regular las webs médicas, considerando como puntos básicos su contenido, aclarando la esponsorización de la misma, y garantizando una total privacidad y confidencialidad ${ }^{18}$.

¿Cómo valorar la calidad de la información obtenida por Internet? El que consulta una página médica debe tener en cuenta los siguientes puntos:

1. Identificación de los autores. La página debe especificar si sus autores son o no profesionales médicos, cual es su afiliación y credenciales, debiendo poder establecer contacto con ellos gracias a e-mail sobre la información proporcionada.

2. Fuentes de información. Deben proporcionar referencias sobre la información médica con links sobre sus fuentes.

3. Confidencialidad. No debe proporcionar información específica sobre un paciente a los que visiten su web, que pueda llevar a su identificación.

4. Actualización. Debe proporcionar datos sobre la fecha de creación de la página médica e incluir las modificaciones posteriores.

5. Información adicional. Debe verificar si existe intereses comerciales, así como si la página está esponsorizada, separando totalmente los anuncios médicos y farmacológicos del contenido científico.

6. Propósito. Debe señalar que la información proporcionada no reemplaza en ningún caso la relación médico-paciente.

Una forma de contrarrestar lo poco útil o nefasta información que Internet pueda proporcionar a los pacientes es que los programas a consultar sean creados por los propios profesionales, ofreciendo incluso muchos de ellos acceso a la información en consejo personalizado. Se establece entonces una especie de "consultorio virtual", con privacidad, -la que puede proporcionar la red-, sobre la información del paciente ${ }^{19}$. El propio Servicio Nacional de Salud de Inglaterra ha creado un programa que proporciona información 
sobre salud y problemas médicos, tanto mediante una página web como por consulta telefónica. En Estados Unidos, la América Doctor (http://www. americasdoctor.com) ha contratado a un grupo de médicos especialistas para que proporcione consejo médico sobre un determinado problema. En España se han multiplicado las páginas web de centros hospitalarios, clínicas privadas y profesionales en ejercicio particular, que se sitúan en esta línea de actuación.

Muchas revistas médicas tradicionales como el Journal of Urology o el Urology están ahora a texto completo en la Red, y algunas, -como la primera de las citadas-, sólo para los socios de la AUA, y otras como Urology de entrada libre gracias a la esponsorización de un laboratorio farmacéuti$\mathrm{co}^{20}$. Pero aparte de estas revistas, las citadas, y otras como JAMA, The Lancet, New England Journal of Medicine, British Medical Journal, etc., existen revistas sólo publicadas "on line", que presentan artículos de revisión sobre un tema concreto o resúmenes de la actualidad médica muy especializada (Medscape, Drs Guider, Uro Health $^{21}$ ), e incluso algunas como Urology Linx ${ }^{22}$ remite diariamente mediante correo electrónico las últimas publicaciones sobre un tema concreto, -cáncer renal, infecciones urinarias...., con posibilidad en algunos casos de acceder mediante un hipertexto o link al artículo completo. Algunas publicaciones solicitan rellenar una hoja sobre información personal para poder acceder al libre acceso de su información, pero ¿quién controla la veracidad de la información remitida?. Si deseamos que el acceso de la página web esté restringida a un grupo de especialistas, ¿cómo certificar que aquel urólogo que dice que vive en Chile por ejemplo, es realmente urólogo y vive en aquel país?.

Cada vez es más frecuente que revistas médicas tradicionales, aparte de poder ser "leídas en el ordenador", publiquen páginas no incluidas en su edición impresa, sólo existentes "on line" y totalmente libres ${ }^{23}$. Generalmente este material suplementario se selecciona en base a su importancia y su trascendencia para los lectores. Y así, al igual que el BUJ International publica "casos prácticos" sólo en Internet, el New England Journal of Medicine $^{24}$, a partir de agosto del 2001 publica en la red artículos que no aparecen en la edición impresa, si bien consta en ellas su presencia en Internet con el hipertexto para conseguirlo.

Internet, tiene además la ventaja que facilita información médica a lugares en los que difícilmente se tiene acceso a muchas de las revistas científicas actualizadas. Y ello es especialmente útil en los países llamados del tercer mundo, en donde el coste de la prensa o libros científicos puede ser prohibitivo. Hace unos 900 años, un gran médico del Islam, -Al-Asuli, escribió una farmacopea médica dividiendo su tratado en dos partes: "enfermedades de los ricos" y "enfermedades de los pobres"25. Aunque el tiempo no ha eliminado en absoluto las diferencias en los tratamientos según la clase social, Internet ha facilitado que, -por lo menos-, el conocimiento y el saber estén al alcance de todos. La información se ha globalizado, siendo ello una gran ventaja, pero asimismo un gran peligro si la información que se recibe no está filtrada, y basada en evidencias ${ }^{26}$.

\section{REFERENCIAS}

1. PIER R.: Cybermedicine. NEJM 1998; 339: 638.

2. EYSENBACH G, RYOUNG SA, DIEPGEN TL.: The impact of informatics. Shopping around the internet today and tomorrow: towards the millennium of cybermedicine. AMJ 1999; 314: 1294.

3. PAL B.: E-mail: contact between doctor and patient. BMJ 1999; 358: 1428.

4. EYSENBACH G, DIEPGEN TL.: Towards quality management of medican information on the internet: evaluation, labelling and filtering of information. BMJ 1998; 37: 1496.

5. JADAD A, GAGLIARDI A.: Rating health information on the internet: navigating to knowledge to Babel. JAMA 1998; 279: 611 .

6. BROWN MS.: Healthcare information seekers aren't typical internet users. Medicine on the net 1998; 4: 1718.

7. PURCELL GP, WILSON P, DELAMOTHE T.: The quality of health information on the internet. BMJ 2002; 324: 557.

8. IMPICCIATORE P.: Reliability of health information for the public on the world wide web: systematic survey on managing fever in children at home. BMJ 1997; 314: 18759.

9. RUTTER T.: Doctors warn of dangers of the Internet. $B M J$ 1998; 317: 1103.

10. GOTLIEB S.: Health information on Internet is often unreliable. BMJ 2000; 321: 136.

11. WHATLING P.: Having non-medical readers of papers on Internet will enhance peer-review. BMJ 1999; 318: 1144.

12. KILEY R.: Does the Internet harm health?. BMJ 2002; 324: 238. 
13. MCLELLAN F.: Like hunger, like thirst: patients, journals and the Internet. The Lancet 1998; 352 (2).

14. Video-Phone in Medicine. http:// www.wired.com/ news/technology/story/19527.html.

15. ITAGAKI M, BERLIN RB, SCHATZ BR.: The rise and fall of E-health: lessons from the first generation of Internet Health care. Medscape tecmed 2002. http://www.medscape.com/viewarticle/431144.

16. SINGH S.: Around every tumor there's a person. BMJ 1998; 316: 56061.

17. GLASS RM et al.: Providing a tool for physicians to educate patients. The JAMA pacient page. JAMA 1998; 279: 13091.

18. WINKER MA et al.: Guidelines for medical and health information sites in the Internet: principles governing AMA web site. JAMA 2000; 283: 1600.

19. PENCHEON D.: NHS Direct: evaluate, integrate or bust. BMJ 1998; 317: 1026.

20. Urology. http:// www.freemedicaljournals.com/ htm/english5.htm. http:// www.medicinedirect. $\mathrm{com} /$ journal/journal?sdid=6105.

21. Mesdcape Urology. http://www.medscape.com/ urologyhome. Urohealth.http://www.urohealth. org. Doctor's Guide. http://www. docguide.com.
22. Urologylinx.http://www.urologylinx.com

23. BJU International. Case reports on line. http://www.blackwell-science.com/products/journals/bjui/cases.

24. New England Journal of Medicine. Internet only publication. Editorial 2001; 345: 365.

25. LOWN B, BUCHAKI F, XAVIER R.: Health information in the developing world. The Lancet 1998; 352 (2). http://www. thelancet.com/search/search/ isa.

26. HERSH WR.: Evidence based medicine and the Internet. Editorial ACP. Journal Clun 5: A, 14, 1996. http:// www. acponline.org/journals/acpjc/jularg96/ jajaedit.htm

Dr. F.J. Solé Balcells

Parque Tilos

Avda. Pedralbes, 37-41, $7^{\mathrm{o}} 1^{\mathrm{a}}$

08034 Barcelona

(Trabajo recibido el 13 noviembre de 2002) 